\title{
THE EFFECT OF AUTOGENOUS, NON-VASCULARIZED PERIOSTEUM TRANSPLANTATION ON ALLOGRAFT BONE GRAFTING FOR LARGE CORTICAL LOAD-BEARING BONE DEFECT - A RABBIT MODEL
}

\author{
AZUHAIRY AZID ${ }^{1,2}$, MOHD ARIFF SHARIFUDIN ${ }^{2,3 *}$, ZULMI WAN², \\ MOHD SHAFIE ABDULLAH ${ }^{4}$ and HASNAN JAAFAR ${ }^{5}$ \\ ${ }^{1}$ Department of Orthopaedics, Penang General Hospital, \\ George Town, Penang, Malaysia \\ ${ }^{2}$ Department of Orthopaedics, School of Medical Sciences, Universiti Sains Malaysia, \\ Kubang Kerian, Kelantan, Malaysia \\ ${ }^{3}$ Faculty of Medicine, Universiti Sultan Zainal Abidin, Kuala Terengganu, \\ Terengganu, Malaysia \\ ${ }^{4}$ Department of Radiology, School of Medical Sciences, Universiti Sains Malaysia, \\ Kubang Kerian, Kelantan, Malaysia \\ ${ }^{5}$ Department of Pathology, School of Medical Sciences, Universiti Sains Malaysia, \\ Kubang Kerian, Kelantan, Malaysia \\ *E-mail: ariffsharifudin@unisza.edu.my
}

Accepted 23 September 2021, Published online 30 November 2021

\begin{abstract}
Segmental bone allografts are widely used in managing large cortical bone defects. To improve host-graft union, the effect of allograft on a large cortical tibial bone defect augmented with a non-vascularized periosteal flap was studied. Twelve mature Australian white rabbits (Oryctolagus cuniculus) were divided into intervention groups and a control group. Bone defects in the intervention groups were treated with segmental allografts wrapped with a non-vascularized periosteal flap. The control group was treated with allograft transplantation alone. Healing was evaluated at the end of the $2^{\text {nd }}, 4^{\text {th }}$, and $6^{\text {th }}$ weeks with plain radiographs, CT scan, and histology. In the intervention groups, the bony union was achieved at both ends of the allografts at the $4^{\text {th }}$ to $6^{\text {th }}$ weeks. Solid callus encasing the whole allograft segments at the end of the $6^{\text {th }}$ week. In the control group, the union did not occur at both ends of the allograft segments even up to the end of the $6^{\text {th }}$ week. No callus formation surrounding the allograft segments. Fragmentation and telescoping of the allograft segment into the medullary cavity of the host were observed. The use of autogenous, non-vascularized periosteal flap modified the healing process of allograft and maintained the integrity of the allograft.
\end{abstract}

Key words: Allograft, bone grafting, large cortical defect, periosteum, union

\section{INTRODUCTION}

The periosteum is a specialized connective tissue that forms a fibrovascular membrane covering the entire surface of bone except for the articular cartilage, ligament at the site of tendon insertions, and the surface of sesamoid bones (Neel, 2003; Allen et al., 2004; Dwek, 2010). It consists of two histological layers; an outer fibrous layer containing blood vessels and nerves supplying the bone, and the inner cambium layer adjacent to the bone, in

\footnotetext{
* To whom correspondence should be addressed.
}

which the cambium (osteogenic) cells reside (Allen et al., 2004; Colnot, 2009; Wang et al., 2017). The cambium cells are osteogenic progenitor cells of mesenchymal origin (Driscoll, 1999; Simon et al., 2003; Colnot, et al., 2012). In children, the cambium layer contributes to the increase of the diameter of the bone during growth (Bisseret et al., 2015). In adults, the cambium cells may be activated after the mechanical stimulus (trauma), infection, and tumorous growth. Under these circumstances, this layer is capable of inducing callus tissue formation and osteogenesis (Simon et al., 2003; Zhang et al., 
2005; Giannoudis et al., 2007; Colnot, 2009; Colnot et al., 2012; Mercurio et al., 2012; Wang et al., 2017). This ability for the cambium cells to differentiate into chondrogenic or osteogenic cells has stimulated interest in their use (Nather \& Goh, 2000; Neel, 2003; Vögelin et al., 2005; Chang \& Knothe-Tate, 2012; Ferretti et al., 2012; van Gastel et al. 2012).

A large segmental bone defect is a common problem faced in orthopedic practice and various surgical options are available for bridging such defect (El-Mowafi et al., 2005). The use of a large segmental bone allograft has become an accepted method of replacing bone defects (McAuliffe, 2003; Vidal et al., 2020). But there are many possible complications associated with this approach. Nonunion, delayed incorporation of graft and failure of an allograft is well observed and documented (Finkemeier, 2001; Betz, 2002; Karaoglu et al., 2003; Zhao \& Zeng, 2003; El-Mowafi et al., 2005; Giannoudis et al., 2007; Vidal et al., 2020). To improve the host-graft union, autogenous vascularized periosteum overlying allografts were used to augment the incorporation of the graft in reconstructive surgery (Karaoglu et al., 2003; Neel, 2003; Barckman et al., 2013).

It is interesting to explore whether the beneficial effects of periosteal grafts transplanted for augmentation of the healing of large segmental bone grafts are the same if the vascularity of the periosteum is not surgically restored during transplantation. The current study was designed to determine whether free autogenous non-vascularized periosteum can survive transplantation in-vivo and to observe its effects on the incorporation of the allograft.

\section{MATERIALS AND METHODS}

A total of 12 mature male New Zealand white rabbits (Oryctolagus cuniculus) aged between 3-5 months old and weight of $2-3 \mathrm{~kg}$ were used in this study. The animals were randomly divided into two groups to form an intervention group (9 rabbits) and a control group (3 rabbits). Two assessment periods were chosen to show the differences in the graft incorporation and healing changes as previous studies indicated histological evidence of fusionhealing changes at around 4-8 weeks (Bax et al., 1999; Nather \& Goh, 2000; Karaoglu et al., 2003; Vögelin et al., 2005). As such, the intervention group was further subdivided into 3 groups of 3 rabbits according to the time of observations (at second, fourth, and sixth weeks, respectively).

Two orthopedic surgeons with previous experience in animal studies and handling performed the administration of the anesthesia and the surgery. All the rabbits were anesthetized using a cocktail of intravenous Ketamine $35 \mathrm{mg} / \mathrm{kg}$ and Xylazine 5 $\mathrm{mg} / \mathrm{kg}$. A prophylactic single dose of antibiotic (Ampicillin $25 \mathrm{mg} / \mathrm{Kg}$ ) was also administered intramuscularly. Under full aseptic technique, a surgical incision was made longitudinally over the anteromedial aspect of the right leg proximal to the ankle joint to expose the tibial bone. A $3 \mathrm{~cm}$ segment of the tibia was exposed and osteotomized at the distal third using a saw with saline irrigation. This segment of bone was kept as fresh frozen allografts in a freezer at $-40^{\circ} \mathrm{C}$. They were stripped bare of their surrounding soft tissues before freezing and to be used for the subsequent rabbits. The defect in the right tibia was replaced with a previously prepared fresh-frozen allograft and stabilized with a Kirschner wire with a diameter of $2 \mathrm{~mm}$ passed retrogradely through the intramedullary canal of the proximal tibia. The wire was cut flushed the bone and buried it subcutaneously.

The bone defects in the rabbits from the control group were treated with allograft transplantation alone. In the intervention group, another similar incision was made on the left leg. The wound was made to expose the periosteum, which was incised and stripped circumferentially away from the bone. The free periosteum of the left tibia was then transferred to the right side and wrapped around the allograft segment and anchored with several sutures. The wounds were irrigated with normal saline and then closed in layers. The leg was immobilized in a Plaster of Paris (POP) cast, extending from above the knee joint to the metatarsal of the foot with the knee kept in 90 degrees flexion and ankle in plantigrade position.

Radiological and histological evaluations were performed at the end of the second, fourth, and sixth weeks. Three rabbits from the intervention group and a rabbit from the control group were selected at each time of observations. They were subjected to plain radiographs and $\mathrm{CT}$ scans of the right tibia with an infusion of intravenous radio-opaque dye. These were performed under general anesthesia. After the radiological evaluations, the rabbits were euthanized by giving an overdose intravascular injection of pentobarbitone $60 \mathrm{mg} / \mathrm{kg}$. The right tibia was harvested by disarticulating through the knee and the ankle joints. The specimen with its surrounding soft tissue was kept submerged in $10 \%$ formalin for hematoxylin and eosin (H\&E) staining and examinations.

\section{RESULTS AND DISCUSSION}

The ideal model of a bone graft should have all the qualities of osteoconduction, osteoinduction, osteogenesis, and structural integrity (Betz, 2002; Finkemeier, 2002; McAuliffe, 2003; Giannoudis et 
al., 2007; Eskandar et al., 2008). An autogenous tricortical bone graft possesses all these, hence, remains as the gold standard option. However, harvesting leads to donor site morbidity and the source is limited (Betz, 2002; Finkemeier, 2002; Giannoudis et al., 2007). Researchers turned to innovating and developing composite grafts that can combine these qualities in a graft model (Giannoudis et al., 2007). The current study was designed in line with this philosophy with the periosteal tissue providing the osteoinductive and osteogenetic properties, whereas the allograft acted as a scaffold (osteoconduction) and structural support. Rabbits were used for this study considering the short duration of fracture healing in them. Only mature male rabbits were used to standardize the gender and to avoid the effects of hormonal fluctuation in female rabbits on bone healing (Arnsdorf et al., 2008; Eskandar et al., 2008).

\section{Two weeks post-transplantation: early healing changes}

All the samples from both groups presented with very mobile osteotomy sites. The plain radiographs showed clear gaps at the osteotomy sites (Figure 1a). In some of the specimens from the intervention group, indistinct callus was seen partially surrounding the proximity of the allograft (Figure 2a). Histological evaluation revealed attempted healing at the osteotomized sites in specimens from both groups (Figure 3a). The transplanted periosteum in the intervention group remained adhered to the allografts with no spaces in between them. The periosteum had lost their cellular components and became thicker, but they maintained their architectural pattern (Figure 3b). These were evidence that the periosteum was undergoing repair and surviving in the new environment. The allografts in both groups showed remnants of cells in the lacuna spaces and there were no surviving cells seen.

Four weeks post-transplantation: restoration of periosteal functions and neovascularization

Plain radiographs of the rabbits from the intervention group showed the presence of callus at the host-graft junctions indicating union at both proximal and distal ends. Callus formations were also seen encasing the allografts segments. However, the fracture lines were still visible (Figure $2 b$ ). CT images with contrast media enhancement confirmed the presence of these encasing callus (Figure 2c). In the control group, the osteotomy sites remained mobile. Some callus was present over the osteotomy sites but not on the allograft segment as seen in the intervention group. The allograft segment appeared smooth without any callus or other soft tissue cover (Figure 1b). Histologically, an excessive healing process was present at both host-graft junctions
(Figure 1d). Callus was seen produced by the survived periosteum in the intervention group as well (Figure 3c). In some of the specimens, the presence of intraosseous callus containing normal osteocytes and new blood vessels was seen in the allograft segments (Figure 3d).

\section{Six weeks post-transplantation: retainment of the integrity of allograft}

All specimens from the intervention group achieved the union of the host-graft junctions with abundant callus formation (Figure 2d). Exuberant callus was also observed encasing the whole allograft segments and present in the intramedullary space, protecting the graft and maintaining its integrity (Figure 3e \& 3f). On the other hand, the allograft segment in the control group was seen fractured and fragmented at the distal host-graft junction leading to telescoping of the graft into the medullary cavity of the host bone despite having abundant surrounding callus (Figure 1e).

\section{Vascularized versus non-vascularized trans- plantation of periosteum}

The use of vascularized periosteum to augment the healing and incorporation of the bone grafts was proven and reported in previous studies (Driscoll, 1999; Neel, 2003; Vögelin et al., 2005; Karaoglu et al., 2013; Barckman et al., 2013). Zhao and Zheng (2003) concluded that large bone defects can be repaired with vascularized tubulate periosteum with or without combination with bone filling materials. The current study was in favor of the results reported by these studies. On the other hand, Askar and Sabuncuoglu (2003) and Eskandar et al. (2008) did not believe that a transplanted periosteum could provide the osteogenetic properties and healing relied directly on the host's recipient bed.

But vascularized transplantation requires microsurgical expertise and takes a longer operational time. Not all surgical institutions can provide the services. In the current study, non-vascularized periosteum was used to augment the healing of allografts. It is technically less demanding than restoring vascularity. The periosteum graft has been proved to be able to initiate osteogenesis in the new environment (Eskandar et al., 2008). But one of the major concerns was to determine whether the free non-vascularized periosteum can survive the transplantation and retained its biological and physiological functions. This was observed in the results obtained from the intervention group of the current study.

The surgical technique of harvesting the periosteum is critical for the success of the transplantation. The cambium layer containing the osteogenic cells delicately adhere to the fibrous layer and can be damaged or left behind on the bone when 

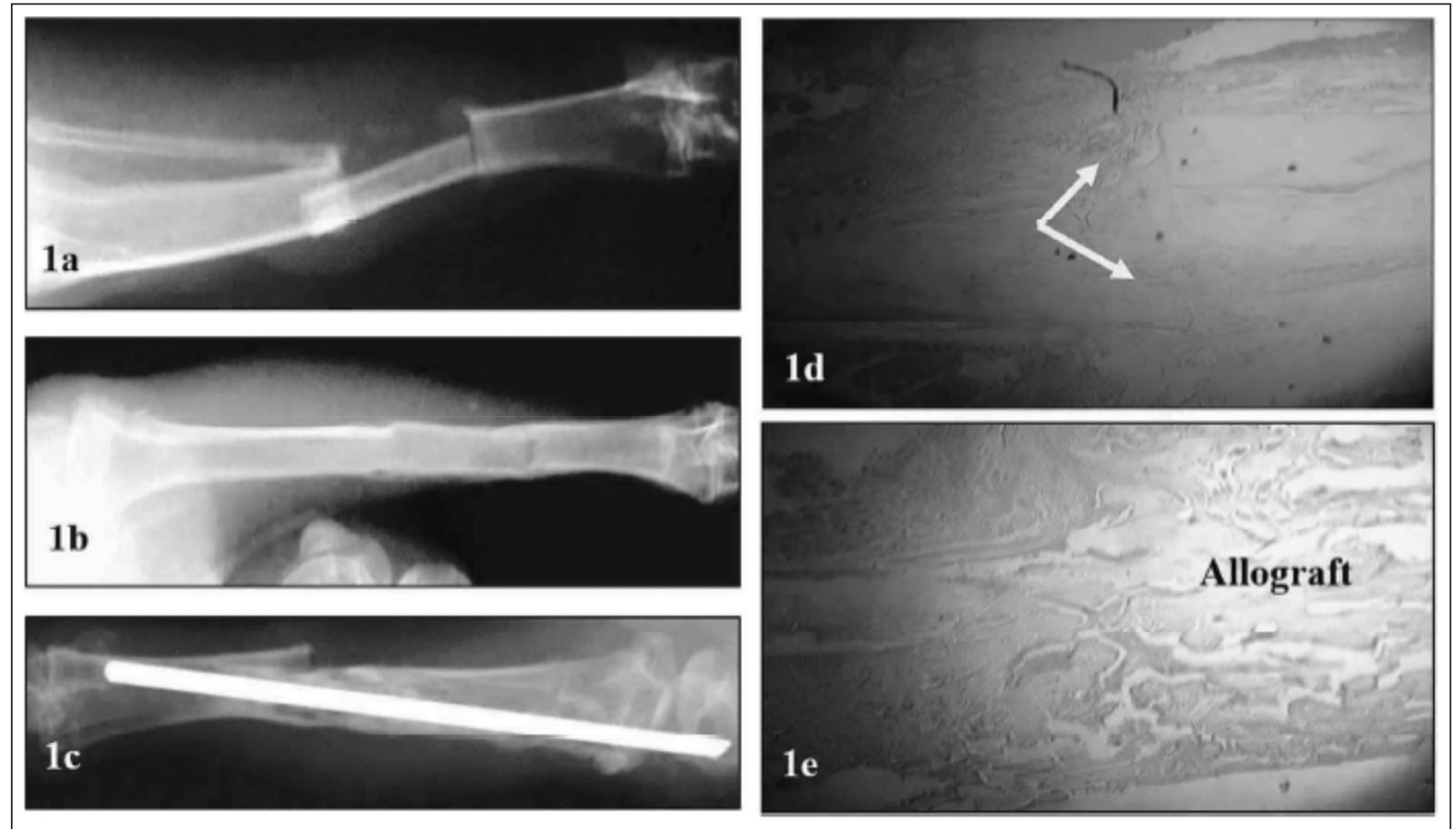

Fig. 1. Evaluation of the right tibiae from the control group. Plain radiographs were taken at the end of (a) 2 weeks, (b) 4 weeks, and (c) 6 weeks. (d) Evidence of the healing process at the host-graft junction started to appear at the end of the fourth week (as shown by the white arrow). (d) At the end of the sixth week, the allograft was seen fractured, fragmented, and telescoped into the medullary cavity of the host bone, despite having abundant surrounding callus.
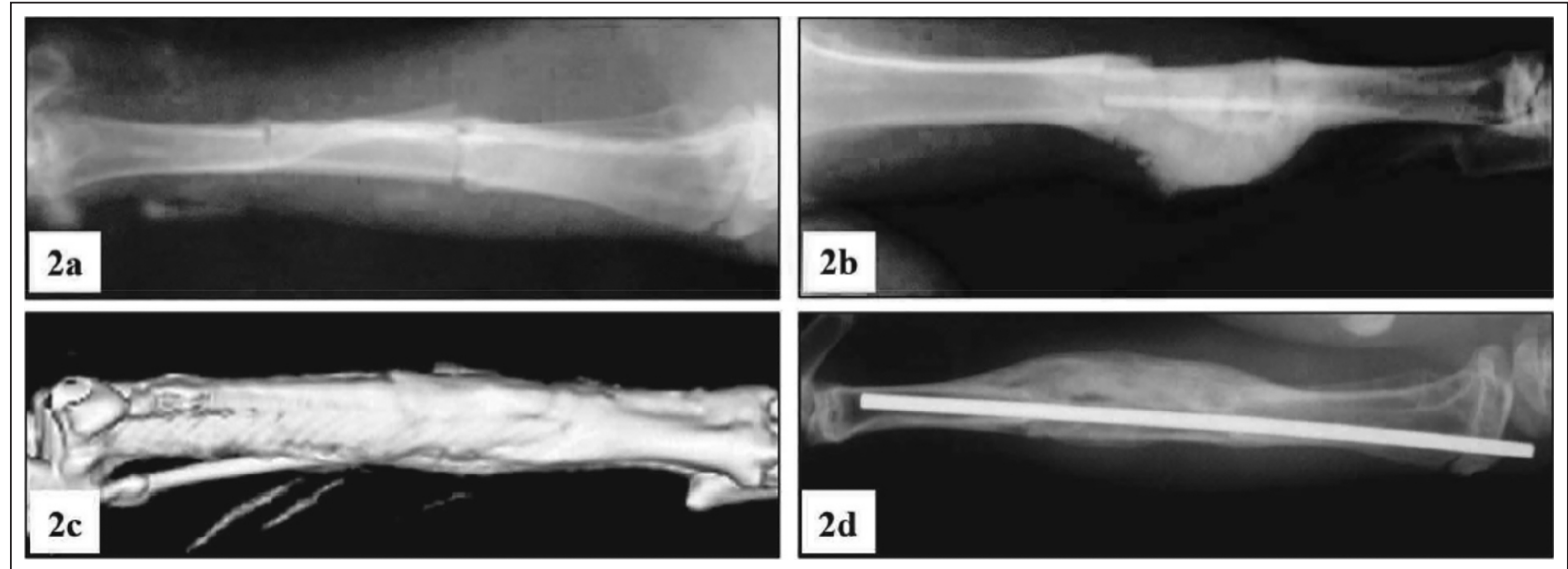

Fig. 2. Radiological evaluation of the right tibiae from the intervention group. (a) At the end of the second week, the osteotomy sites were still visible with minimal callus surrounding the allograft segment. (b) An abundance of callus was seen at both ends and around the allograft at the end of the fourth week on (b) plain radiograph and (c) 3D reconstructed CT image. (d) Bony union was evident at the host-graft junction with exuberant callus was seen encasing the whole of the allograft segment at the end of the sixth week.

the periosteum is harvested (Brownlow et al., 2000; Driscoll \& Fitzsimmons, 2000; Simon et al., 2003; Zhang et al., 2005; Colnot, 2009; Dwek, 2010; Colnot et al., 2012; Mercurio et al., 2012; Bisseret et al., 2015). Technical precautions should be taken during periosteal harvesting and transplantation.

\section{CONCLUSION}

The free non-vascularized periosteum survived the in-vivo transplantation and retained its beneficial biological and physiological functions. The allograft healing and host-junction incorporation was 


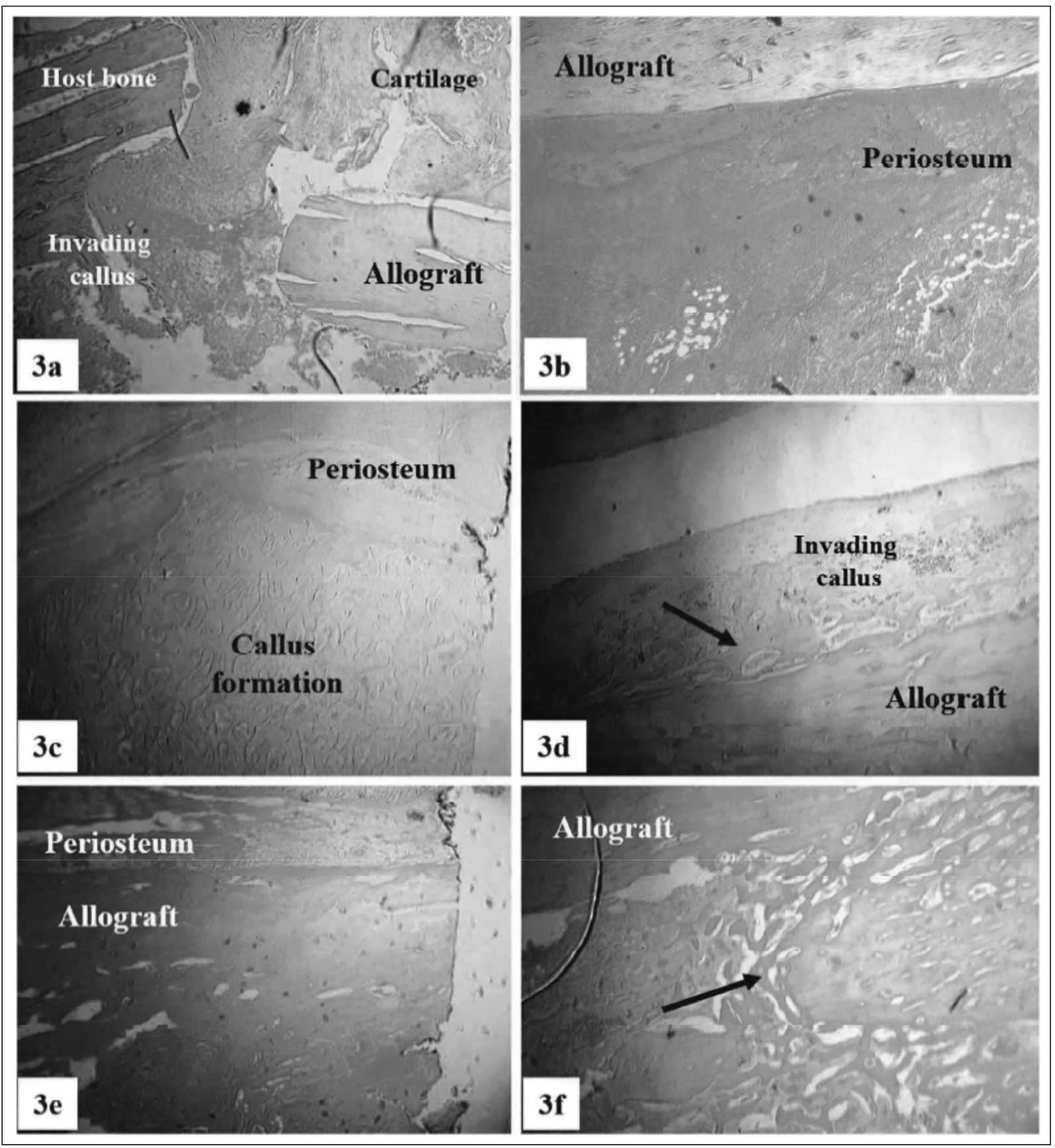

Fig. 3. Histological evaluation of the right tibiae from the intervention group. At the end of the second week, (a) attempted healing was visualized at the host-graft evident by the presence of callus, and (b) the periosteum remained to adhere to the allograft and became thickened, indicating that it was undergoing repair and surviving in the environment. At the end of the fourth week, (c) the survived periosteum started to produce callus, and (d) evidence of intraosseous callus formations with neovascularization (as shown by the black arrow). (e and f) The integrity of the allograft was maintained with parts of the allograft being replaced by new bone (as shown by the black arrows.

modified by the transplanted periosteum by hastening the callus formation at the host-graft junctions. The integrity of the allograft was protected by the formation of callus encasing the graft as well as the presence of intramedullary callus.

\section{ACKNOWLEDGMENTS}

The authors would like to acknowledge Universiti Sains Malaysia (USM) for the financial support provided under the USM Short Term Grant (No.: 304/ PPSP/6131374). 


\section{REFERENCES}

Allen, M.R., Hock, J.M. \& Burr, D.B. 2004. Periosteum: biology, regulation, and response to osteoporosis therapies. Bone, 35(5): 1003-1012.

Arnsdorf, E.J., Jones, L.M., Carter-Ogita, M., Rached, M.T., Dworakowski, E., Bilezikian, J.P. \& Kousteni, S. 2008. Differentiation and proliferation of periosteal osteoblast progenitors are differentially regulated by estrogens and intermittent parathyroid hormone administration. Endocrinology, 149(11): 5713-5723.

Askar, I. \& Sabuncuoglu, B.T. 2003. Prefabrication of periosteal graft alone or with oxidised cellulose: an experimental study. Acta Chirurgiae Plasticae, 45(1): 3-7.

Barckman, J., Baas, J., Sorensen, M., Bechtold, J.E. \& Soballe, K. 2013. Periosteal augmentation of allograft bone and its effect on implant fixation an experimental study on 12 dogs. The Open Orthopaedics Journal, 7: 18-24.

Bax, B.E., Wozney, J.M. \& Ashhurst, D.E. 1999. Bone morphogenetic protein-2 increases the rate of callus formation after fracture of the rabbit tibia. Calcified Tissue International, 65(1): 83-89.

Betz, R.R. 2002. Limitations of autograft and allograft: new synthetic solutions. Orthopedics, 25(5 Suppl): 561-570.

Bisseret, D., Kaci, R., Lafage-Proust, M.H., Alison, M., Parlier-Cuau, C., Laredo, J.D. \& Bousson, V. 2015. Periosteum: Characteristic imaging findings with emphasis on radiologic-pathologic comparisons. Skeletal Radiology, 44(3): 321-338.

Brownlow, H.C., Reed, A., Joyner, C. \& Simpson, A.H.R.W. 2000. Anatomical effects of periosteal elevation. Journal of Orthopaedic Research, 18(3): 500-502.

Chang, H. \& Knothe-Tate, M.L. 2012. Concise review: the periosteum: Tapping into a reservoir of clinically useful progenitor cells. Stem Cells Translational Medicine, 1(6): 480-491.

Colnot, C. 2009. Skeletal cell fate decisions within periosteum and bone marrow during bone regeneration. Journal of Bone and Mineral Research, 24(2): 274-282.

Colnot, C., Zhang, X. \& Knothe-Tate, M.L. 2012. Current insights on the regenerative potential of the periosteum: Molecular, cellular, and endogenous engineering approaches. Journal of Orthopaedic Research, 30(12): 1869-1878.

Driscoll, S.W. 1999. Articular cartilage regeneration using periosteum. Clinical Orthopaedics, 367: S186-S203.
Driscoll, S.W. \& Fitzsimmons, J.S. 2000. The importance of procedure specific training in harvesting periosteum for chondrogenesis. Clinical Orthopaedics, 380: 269-278.

Dwek, J.R. 2010. The periosteum: what is it, where is it, and what mimics it in its absence?. Skeletal Radiology, 39(4): 319-323.

El-Mowafi, H., Elalfi, B. \& Wasfi, K. 2005. Functional outcome following treatment of segmental skeletal defects of the forearm bones by Ilizarov application. Acta Orthopaedica Belgica, 71(2): 157-162.

Eskandar, H., Zulmi, W., Suzina, A. \& Samsudin, A.R. 2008. The use of coral as a graft in a large cortical bone defect. Nature Precedings, 3: 2264.

Ferretti, C., Borsari, V., Falconi, M., Gigante, A., Lazzarini, R., Fini, M., Di Primio, R. \& MattioliBelmonte, M. 2012. Human periosteum-derived stem cells for tissue engineering applications: the role of VEGF. Stem Cell Reviews and Reports, 8(3): 882-890.

Finkemeier, C.G. 2002. Bone-grafting and bone-graft substitutes. Journal of Bone and Joint Surgery (American Volume), 84(3): 454-464.

Giannoudis, P.V., Einhorn, T.A. \& Marsh, D. 2007. Fracture healing: the diamond concept. Injury, 38(Supp 4): S3-S6.

Karaoglu, S., Baktir, A., Kabak, S. \& Arasi, H. 2003. Experimental repair of segmental bone defects in rabbits by demineralized allograft covered by free autogenous periosteum. Injury, 33(8): 679-683.

McAuliffe, J.A. 2003. Bone graft substitutes. Journal of Hand Therapy, 16(2): 180-187.

Mercurio, A.D., Motta, T., Green, E., Noble, G., Hart, R.T. \& Allen, M.J. 2012. Effects of extensive circumferential periosteal stripping on the microstructure and mechanical properties of the murine femoral cortex. Journal of Orthopaedic Research, 30(4): 561-568.

Nather, A. \& Goh, J.C.H. 2000. Biochemical strength of large diaphyseal deep frozen allografts. Cell and Tissue Banking, 1(3): 201-206.

Neel, M. 2003. The use of periosteal replacement membrane for bone graft containment at allograft-host junctions after tumor resection and reconstruction with bulk allograft. Orthopedics, 26(5): S587.

Simon, T.M., Sickle, D.V.C., Kunishima, D.H. \& Jackson, D.W. 2003. Cambium cell stimulation from surgical release of the periosteum. Journal of Orthopaedic Research, 21(3): 470-480.

van Gastel, N., Torrekens, S., Roberts, S.J., Moermans, K., Schrooten, J., Carmeliet, P., Luttun, A., Luyten, F.P. \& Carmeliet, G. 2012. Engineering vascularized bone: Osteogenic and proangiogenic potential of murine periosteal cells. Stem Cells, 30(11): 2460-2471. 
Vidal, L., Kampleitner, C., Brennan, M.A., Hoornaert, A. \& Layrolle, P. 2020. Reconstruction of large skeletal defects: Current clinical therapeutic strategies and future directions using 3D printing. Frontiers in Bioengineering and Biotechnology, 8:61.

Vögelin, E., Jones, N.F., Huang, J.I., Brekke, J.H. \& Lieberman, J.R. 2005. Healing of a critically-sized defect in the rat femur with use of a vascularized periosteal flap, a biodegradable matrix, and bone morphogenetic protein. Journal of Bone and Joint Surgery. (American Volume), 87(6): 1323 1331.

Wang, T., Zhang, X. \& Bikle, D.D. 2017. Osteogenic differentiation of periosteal cells during fracture healing. Journal of Cellular Physiology, 232(5): 913-921.
Zhang, X., Xie, C., Lin, A.S., Ito, H., Awad, H., Lieberman, J.R., Rubery, P.T., Schwarz, E.M., O'Keefe, R.J. \& Guldberg, R.E. 2005. Periosteal progenitor cell fate in segmental cortical bone graft transplantations: implications for functional tissue engineering. Journal of Bone and Mineral Research, 20(12): 2124-2137.

Zhao, J.Z. \& Zeng, B.F. 2003. Treatment of large bone defect of radius with vascularised tubulate periosteum graft and bone filling material in rabbits. National Library of Medicine, 17(1): 912. 
